\title{
A Heuristic Tool for Exposure Reduction in Indoor Wireless Networks
}

\author{
David Plets*, Wout Joseph, Kris Vanhecke, Luc Martens \\ Dept. of Information Technology, Ghent University/IBBT \\ Ghent, Belgium \\ Email: david.plets@intec.ugent.be
}

\begin{abstract}
A heuristic indoor network planner for exposure calculation and optimization in wireless networks is developed. The model for the electric-field strength in the vicinity of an access point is presented and the WiFi networks are optimized in order not to exceed a maximal electric-field strength at a certain separation from the access points. The influence of the maximally allowed field strength and the assumed minimal separation between the access point and the human is assessed for a typical office building.
\end{abstract}

\section{INTRODUCTION}

Due to the increased popularity of indoor wireless networks, many software tools have been developed for the prediction of the received signal quality and the network performance. The heuristic WHIPP (WiCa Heuristic Indoor Propagation Prediction) tool, based on the dominant path model is able to design a WiFi network for a given coverage requirement with a minimal number of access points (APs) and has been created in cooperation with usability experts [1]. It is implemented as a web service with a Java engine that allows the user to draw or import the ground plan of a building and predict and optimize the coverage in the different rooms on a floor level. In the meanwhile, the enormous increase of wireless communication makes it necessary to characterize the exposure of people due to electromagnetic fields at RF (radio-frequency) frequencies and to investigate the exposure of the general public to wireless telecommunication systems. International guidelines such as ICNIRP [2] have been developed and authorities and countries have implemented laws and norms to limit human exposure. Some cities (e.g., Salzburg, Paris,...) even have their own specific guidelines. This indicates the need for accurate exposure calculations. Therefore, an exposure prediction and optimization module has been developed for and added to the WHIPP tool. In this paper, this module will be presented. A model for the electric-field strength in the vicinity of an AP is constructed and validated with simulations and measurements. An exposure map will be shown for a specific exposure requirement in an office building. The influence of the maximally allowed field strength and the assumed minimal separation between the AP and the human will be assessed for a typical office building and for exposure limits (or recommendations) in different regions in the world.

\section{EXPOSURE MODEL}

It will be investigated whether the free-space far-field model is also applicable to the near-field. Fig. 1 shows the electric- field strength of a half-wavelength dipole at $2400 \mathrm{MHz}$ with an EIRP (Equivalent Isotropically Radiated Power) of $20 \mathrm{dBm}$ as a function of the separation from the dipole, according to an FDTD (Finite-Difference Time-Domain) simulation between 3.5 and $50 \mathrm{~cm}$ from the dipole. Fig. 1 also shows the measured electric-field strength as a function of the separation from a DLink DI-624 AirPlusXtremeG AP with an EIRP of $20 \mathrm{dBm}$ and a duty cycle of $100 \%$. The figure shows that the electricfield strength model obtained from far-field free-space pathloss conversion is a very good approximation for both the measured and simulated near-field electric-field strength. The higher measured deviations at larger separations may be due to wall reflections. The results obtained in this section show that the use of the converted free-space loss model is a feasible choice for the exposure model near the AP (between $10 \mathrm{~cm}$ and $3 \mathrm{~m}$ from the AP).

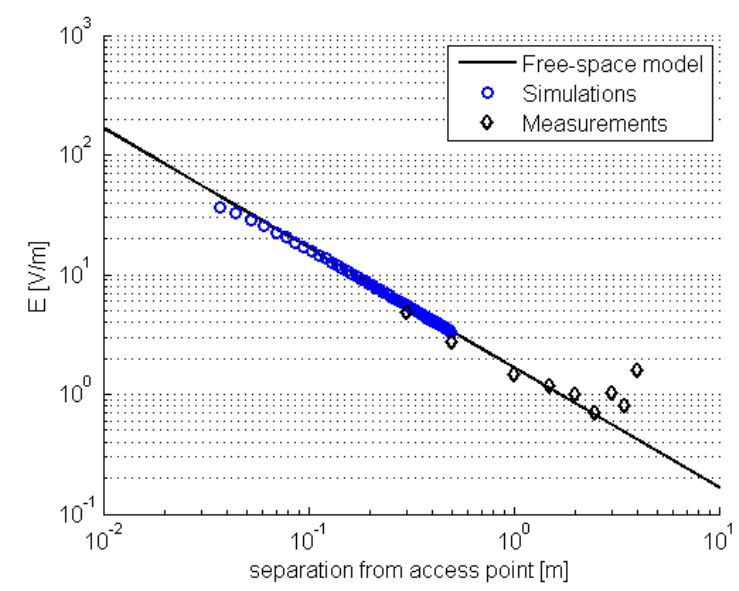

Fig. 1. Measured electric-field strength around a DLink DI-624 AP with an EIRP of $20 \mathrm{dBm}$, electric-field strength around a dipole with an EIRP of 20 $\mathrm{dBm}$ according to FDTD simulations, and free-space model.

\section{RESULTS}

Fig. 2 shows the ground plan of an office building, for which we intend to limit the human exposure. The configuration is optimized for 'HD video' access in the entire building, except in the shaded areas, where no coverage is needed (kitchen, toilet, elevator,...). For a maximal electric field of $5 \mathrm{~V} / \mathrm{m}$ at a separation of $10 \mathrm{~cm}$ from the AP, 10 APs (with an EIRP of $9 \mathrm{dBm}$ ) are needed. The figure also shows the resulting 


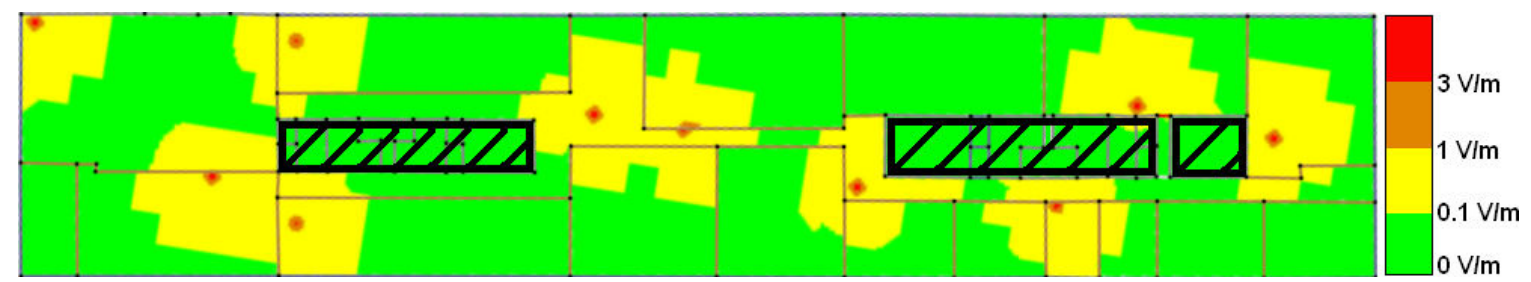

Fig. 2. Ground plan of the building that is optimized for a maximal electric field of $5 \mathrm{~V} / \mathrm{m}$ at a separation of $10 \mathrm{~cm}$ from the AP and indication of electric-field strength (red: $\mathrm{E}>3 \mathrm{~V} / \mathrm{m}$, orange: $3 \mathrm{~V} / \mathrm{m} \geq \mathrm{E}>1 \mathrm{~V} / \mathrm{m}$, yellow: $1 \mathrm{~V} / \mathrm{m} \geq \mathrm{E}>0.1 \mathrm{~V} / \mathrm{m}$, green: $0.1 \mathrm{~V} / \mathrm{m} \geq \mathrm{E}$ ). The APs are located in the red/orange zones. The shaded areas indicate where no wireless coverage is required.

exposure map. The APs are not indicated in the figure, but it is clear that their locations correspond to the red/orange dots where the exposure is the highest. Obviously, the electric-field strength decreases when moving away from the APs.

Fig. 3 shows the number of APs needed to provide the required coverage on the building floor, as a function of the maximal limit $\mathrm{E}_{\max }$ for different minimal separations from the AP, ranging from $10 \mathrm{~cm}$ to $3 \mathrm{~m}$. When, at a fixed separation from the AP, the maximally allowed exposure limit $\mathrm{E}_{\max }$ increases, a higher EIRP is allowed for the APs, leading to a lower total number of APs needed to cover the building floor. At high $\mathrm{E}_{\max }$ values, the number of APs becomes constant (3 APs for this configuration), since the EIRP at $2.4 \mathrm{GHz}$ is limited at $20 \mathrm{dBm}$. Alternatively, if the assumed minimal separation from an AP decreases (for a fixed value of $E_{\max }$ ), the maximally allowed EIRP decreases and more APs are needed to cover the building floor. Fig. 2 corresponds with $\mathrm{E}_{\max }=5 \mathrm{~V} / \mathrm{m}$ at $10 \mathrm{~cm}$ and is indicated in Fig. 3 .

Table I shows the number of APs needed to cover the building floor depicted in Fig. 2, for different Rx-Ap separations and for exposure limits (or recommendations) in different countries. The different exposure limits cause large differences in the required number of APs (and their maximal EIRP). It is clear that for very restrictive limits (low $\mathrm{E}_{\max }$ values), network planning becomes extremely difficult (e.g., Salzburg).

\begin{tabular}{|c|c|c|c|c|c|c|}
\hline \multicolumn{2}{|c|}{ \# Access points } & \multicolumn{5}{|c|}{ Separation between $\mathrm{Rx}$ and $\mathrm{AP}[\mathrm{cm}]$} \\
\hline Region & Limit $[\mathbf{V} / \mathbf{m}]$ & 10 & 30 & 50 & 100 & 300 \\
\hline Salzburg $^{1}$ & 0.02 & $>75$ & $>75$ & $>75$ & $>75$ & $>75$ \\
\hline Wallonia $^{12}$ & 3 & 16 & 5 & 4 & 3 & 3 \\
\hline Flanders $^{12}$ & 4.48 & 11 & 4 & 3 & 3 & 3 \\
\hline Italy $^{1}$ & 6 & 6 & 3 & 3 & 3 & 3 \\
\hline China $^{1}$ & 12 & 4 & 3 & 3 & 3 & 3 \\
\hline & (ICNIRP) 61 & 3 & 3 & 3 & 3 & 3 \\
\hline
\end{tabular}

TABLE I

NUMBER OF APS NEEDED TO COVER THE BUILDING FLOOR DEPICTED IN FIG. 2, FOR EXPOSURE LIMITS IN DIFFERENT COUNTRIES.

1: FROM HTTP://WWW.WHO.INT/DOCSTORE/PEH-EMF/EMFSTANDARDS

2 : WALLONIA AND FLANDERS ARE REGIONS IN BELGIUM

\section{Conclusion}

A heuristic indoor network planner for exposure calculation and optimization in wireless networks is developed. The model

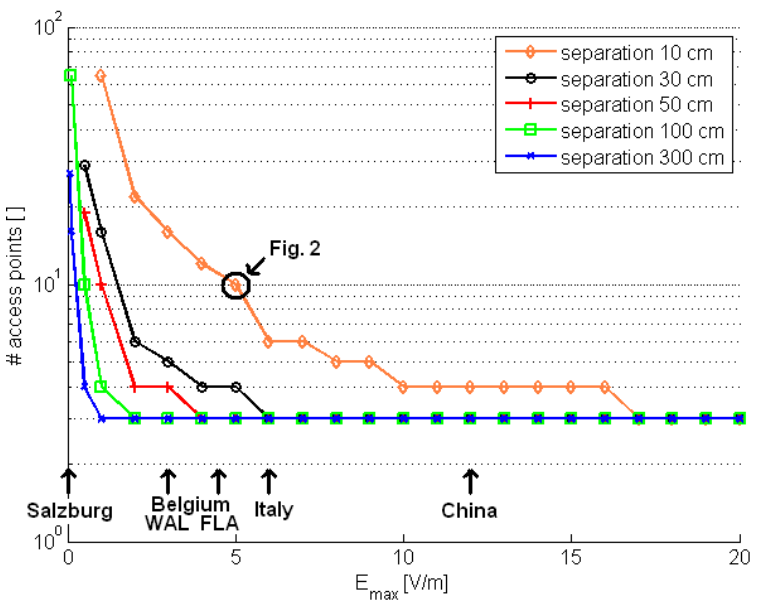

Fig. 3. Number of APs needed to provide the required coverage on the building floor, as a function of the maximal limit $\mathrm{E}_{\max }$ for different minimal separations from the AP, and indication of limits or recommendations in different regions.

for the electric-field strength in the vicinity of an AP is presented and the WiFi network in an office building is optimized in order not to exceed a maximal electric-field strength at a certain separation from the APs. It is shown that higher allowed exposure limits and higher separations between the AP and the human, allow higher transmit powers and hence, a lower number of APs is required to provide coverage. The required number is compared for exposure limits in different countries in the world. Future research includes exposure reduction in heterogeneous networks (where the human is simultaneously exposed to radiation from different wireless technologies (e.g., LTE (Long-Term Evolution) and WiFi).

\section{ACKNOWLEDGEMENT}

This work was supported by the IWT-SBO SymbioNets project. W. Joseph is a Post-Doctoral Fellow of the FWO-V (Research Foundation-Flanders).

\section{REFERENCES}

[1] D. Plets, W. Joseph, K. Vanhecke, E. Tanghe, and L. Martens, "Coverage Prediction and Optimization Algorithms for Indoor Environments," EURASIP Journal on Wireless Communications and Networking, Special Issue, 2012, accepted.

[2] ICNIRP, "Guidelines for limiting exposure to time-varying electric, magnetic, and electromagnetic fields (up to $300 \mathrm{GHz}$ )," Health Physics, vol. 74, no. 4, pp. 494-522, Apr. 1998. 